\title{
Ley chatarra sobre alimentación saludable
}

\section{Oscar Sumar A.}

En el presente artículo, el Profesor Oscar Súmar, realiza un crítico análisis de la regulación sobre comida chatarra. Analiza cada una de las justificaciones a la norma, y llega a la conclusión que este propósito es "irracional con efectos para todos".

Profesor a tiempo completo en la Facultad de Derecho de la UP. Abogado. Master en Derecho por UC Berkeley. 


\section{Ley chatarra sobre alimentación saludable}

Conozco personas que no comen un animal sin antes saber si lo trataron bien antes de sacrificarlo, para ser cocinado. También conozco personas que no toman agua que tenga elementos minerales. Estos podrían ser calificados como refinamientos de la dieta que están lejos de ser las necesidades básicas de las personas. Para la gran mayoría de personas en el Mundo, sus preocupaciones en relación a la comida son mucho más primarias: ¿tengo suficiente comida para sobrevivir?

En ese escenario, el Gobierno publicó una norma para desincentivar el consumo de comidas "procesadas" y para eliminar el consumo de "grasas trans". Sin duda, estas normas no son tan "sofisticadas" como preocuparse de si trataron bien al pollo antes de matarlo; pero comparten algo de su frivolidad, en un contexto donde las personas buscan qué comer mañana. Digo esto porque, en Perú, la obesidad no es precisamente un problema relacionado a la pobreza, ni al consumo de la comida procesada que será afectada por esta norma.

\section{¿Cuál es la justificación de esta norma?}

\section{Externalidades no internalizadas}

Nunca nadie ha muerto de second hand eating. Sin embargo, los propulsores de normas como las de la alimentación saludable argumentan que la comida chatarra causa externalidades a la sociedad, debido a que el Estado necesita recaudar más tributos para poder pagar las cuentas médicas de los obesos. Además, la obesidad -y las enfermedades relacionadas a la obesidad- reduciría la productividad de las personas.

Ambos criterios son erróneos. Por un lado, ninguno es realmente una externalidad. Los gastos son asumidos por el Estado, por las empresas o por los obesos; por propia iniciativa. Si el Estado paga las cuentas es porque ha decidido hacerlo. Sin un gordo es poco productivo, entonces él deja de ganar dinero, sea por descuentos directos o por reducción de su empleabilidad.

Fuera de esto, aun cuando considerásemos que los gastos médicos son realmente una externalidad, no es tan claro que los obesos causen más gastos al erario que los flacos. La gente decía que los fumadores ocasionaban más gastos al Estado que los no-fumadores. Esta afirmación fue puesta a prueba 
por el profesor de regulación Kip Viscusi ${ }^{1}$. Él descubrió que, en realidad, los fumadores significaban una reducción de gastos para el Estado. Esto es porque morían más jóvenes, entonces tenían menos enfermedades asociadas a la vejez y recibían menos anos de pensión.

\section{La moral: el hombre ideal}

La otra justificación es "moralista". Parte de creer que un ser humano esbelto, sano y productivo es intrínsecamente mejor que uno mofletudo, enfermizo y flojo. Parece ser, por lo menos, la opinión del presidente Humala, que ha destacado la necesidad de tener un Estado "esbelto" en contraposición a una "gordo", en diversos discursos públicos. Esta idea del "hombre ideal", ¿es suficiente para impulsar la maquinaria estatal? Sinceramente, creo que no, pero estoy partiendo desde una perspectiva liberal aquí. Creo que el Estado no debería tener opiniones morales "fuertes". Es decir, que solo deberían concentrarse en los aspectos morales esenciales, como el respeto a la vida, pero no en detalles como la manera en la que deberíamos vivir nuestras vidas.

\section{Irracionalidad}

Se dice que los consumidores no son suficientemente racionales para saber que la comida chatarra les causa daño. Asumamos que esto es cierto. Existe evidencia de que las personas toman decisiones de consumo inconscientes y que la publicidad explota esto para persuadirnos de adquirir un bien. Si esto es así, es cierto para todos los productos, no solo para la comida chatarra. Si el fundamento para restringir la publicidad de la comida chatarra es que somos irracionales, entonces deberíamos prohibir (o restringir) toda la publicidad. Pero resulta que -por más que nos quieren hacer pensar lo contrario- la publicidad es un bien, que nos permite adquirir mejores productos a mejores precios, al intensificar la competencia entre empresas. Si se prohibiera la publicidad, menos personas intentarían "jugar con nuestras mentes", pero también compraríamos peores productos a precios más altos. La pregunta es, ¿qué preferimos?

\section{Asimetría informativa}

Ligado a lo anterior, también se ha argumentado que las personas no tienen suficiente información acerca de lo dañina que es la comida chatarra. Esto es una especulación. Nadie se ha tomado el trabajo de verificarlo. A mi me suena

VISCUSI, Kip. Smoke-Filled Rooms: A Postmortem on the Tobacco Deal. New York: Free Press. 1998 
a cuento. Si pones a una persona una ensalada y una hamburguesa, todos saben -o creen- que la ensalada es más sana.

En relación a este punto, se dice que las personas no sabrían el contenido de las comidas, ni que algunas pueden ser dañinas o cuán dañinas son. Así, se requeriría o prohibir algunos productos particularmente dañinos u obligar a las empresas a revelar información.

Esta idea, sin embargo, además de ser una mera especulación se olvida de varios puntos:

(i) No necesitamos, ni queremos, tener toda la información. En Economía se utiliza el concepto de "ignorancia racional" para designar el punto en el cual obtener información adicional seria irracional por significar más costos que beneficios. Algunas veces se obliga a revelar información, pese a que no existe evidencia de que los consumidores quieran esa información. Es el caso de la obligación de indicar que los productos son "transgénicos". Muchas veces las personas no saben que significa y no les interesa, pese a lo cual es muy importante para unos cuantos congresistas que esta información se divulgue. Estar informado es un "derecho" y eso es aparentemente- justificación suficiente para dejar de lado el sentido común y la racionalidad.

(ii) Los consumidores más informados pueden elevar la calidad o reducir el precio para todos. Es el llamado "arbitraje" de información. En otras palabras «Si los vendedores no pueden distinguir fácilmente entre los consumidores informados y los desinformados, ellos no pueden explotar a los desinformados cobrándoles un precio más alto» ${ }^{2} 0$ dándoles una calidad inferior.

(iii) El mercado, a través del precio y la publicidad, da suficiente información a los consumidores. Aunque estos no conozcan el mismo nivel de detalle que los expertos, tienen información que funciona como sustituto y que

2 Eric Posner. Economic Analysis of Contract Law After Three Decades: Success or Failure?. En: Yale Law Journal 112. 2002. Página 844.

Traducción libre de: «If sellers cannot easily distinguish informed and uninformed consumers, they cannot exploit the latter by charging them a higher price». 
tiene un efecto similar al determinar la calidad de los productos ${ }^{3}$ (Rodríguez y Sumar 2013).

\section{La redistribución: ¿pobreza asociada a la obesidad?}

En San Francisco, la primera vez que fui a un McDonalds quedé sorprendido porque el lugar estaba lleno de homeless (vagabundos). Meses después, McDonalds eliminó su One Dollar Menu. Adivinen quién protestó... Así es, la asociación de vagabundos de San Francisco ${ }^{4}$. Ellos dijeron que, el haber quitado el menú de un dólar era el equivalente a que la municipalidad de San Francisco hubiera sacado una ordenanza prohibiéndoles entrar a McDonalds.

Independientemente de la anécdota, lo cierto es en un país como Estados Unidos, comer mal equivale a comer comida chatarra. ¿Esto es cierto en Perú? ¿Cuándo fue la última vez que vio a un vagabundo comprando una hamburguesa en McDonalds?

Además de esto, si fuera el caso que los pobres comen en McDonalds, ¿se justificaría prohibir o dificultar su obtención como una política social? ¿Prohibir las "malas" opciones incrementa las "buenas"? En España la gente comenzó a comer de los tachos de basura debido a la crisis. El Gobierno decidió cerrar los tachos con candados. Felizmente también decidió regalar comida. Ojala eso haya ido bien, pero cerrar los tachos por sí mismo no ayuda a los pobres. Aumentar, mediante regulación, el precio de los alimentos; termina perjudicando a los más pobres.

Existen personas en el Mundo que tienen la posibilidad de preocuparse si el agua que toman tiene o no contenido mineral o si trataron bien al pollo antes de sacrificarlo. Esas personas realmente están dispuestas a invertir parte de su dinero en estas preferencias. Es hasta cierto punto frívolo pensar que una persona pobre puede ver la comida como algo más que la posibilidad de supervivencia. Una comida alta en calorías es una elección bastante inteligente desde el punto de vista de un pobre. Piense alguna vez que se haya muerto de hambre durante toda la mañana/tarde y solo llegue a comer en la noche. ¿Le provocó una ensalada? Aplique eso a la vida diaria y multiplíquelo por los 365

3 Gustavo Rodriguez y Oscar Sumar. Una Demostración empírica de la sabiduría del mercado: sobre la percepción de la calidad de las facultades de Derecho en Perú. (Inédito).

$4 \quad$ Ver: http://www.sfgate.com/restaurants/article/Hassle-in-Haight-over-McDonald-s-menuchange-3175719.php 
días de un año, la próxima vez que quiera criticar los hábitos alimenticios de una persona con escasos recursos. En este sentido, se ha dicho que:

«La realidad es que la gente que no tiene suficiente dinero (o el equipamiento o capacidad de almacenamiento) para comprar y preparar comida decente en cantidades decentes no puedes ( $y$ no debe) ser forzada a preocuparse por los detalles más finos sobre nutrición. Esto, porque tener comida suficiente es siempre nuestra prioridad.

Es por eso que Ellyn Satter [...] creó la Jerarquía de Necesidades Alimenticias. La jerarquía es como sigue:

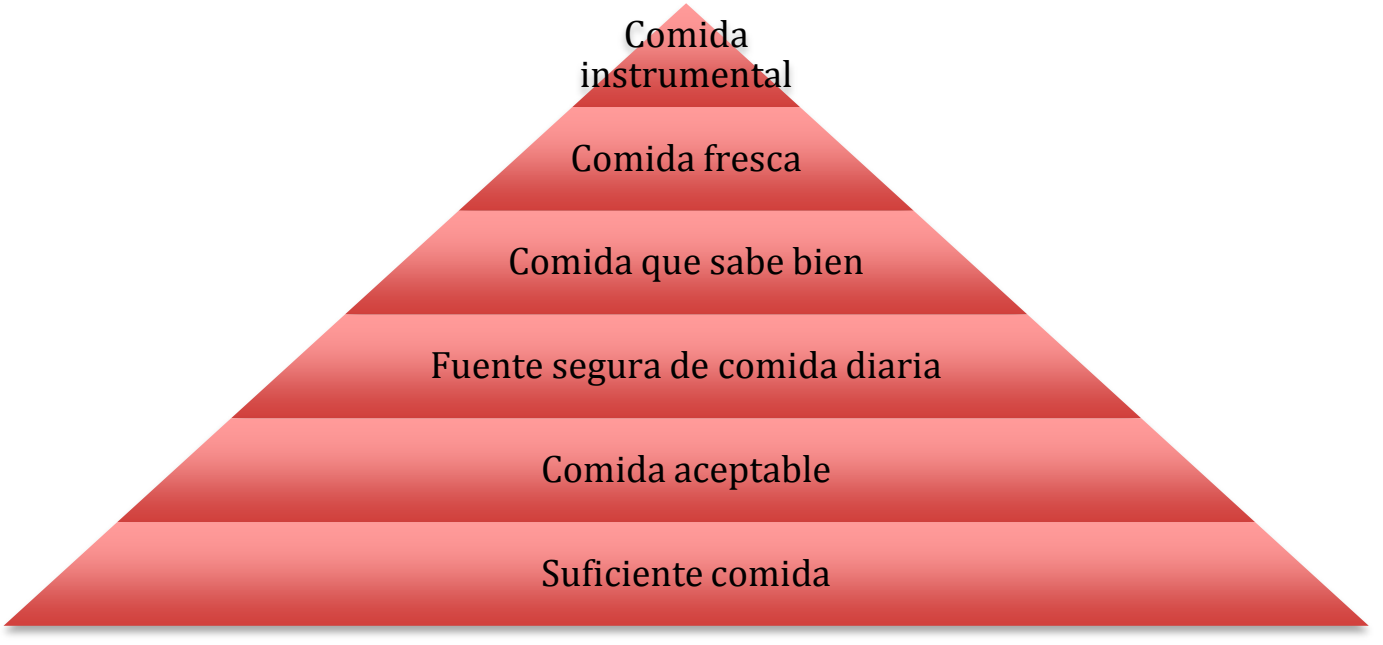

La idea es que, antes de preocuparnos por cosas como la nutrición (esto es, "comida instrumental") primero tenemos que TENER comida. Suficiente comida. Consistentemente. $Y$ tiene que ser aceptable para nosotros (lo que, para algunas personas, puede significar no venir de la basura, o cumplir con ciertos estándares de 
preparación) y tiene que saber razonablemente bien. Un poco de variedad es buena, también» 5 .

¿Suficientemente claro?

\section{Nivel de consumo distinto del óptimo}

Otra cosa que se podría decir -y que constituiría la única justificación económica propiamente de una norma como ésta- es que el consumo de comida chatarra en Perú es mayor al óptimo. ¿Imagina una Policía del Trámite con miles de agentes y penas de muerte aplicadas instantáneamente por jueces/policías? ¿Imagina un país sin carros? ¿Imagina a una Agencia Estatal del Matrimonio o de la Amistad? ¿Imagina un país donde hacer ejercicios sea un deber tanto como lo es respetar la vida de los demás? Probablemente todas esas medidas serían necesarias -aunque no suficientes- para asegurar que vivamos en un país sin corrupción; accidentes de tránsito; discriminación o enfermedades asociadas al sobrepeso... Todos estos son percibidos como males sociales, pero, ¿son justificaciones para la regulación? Desde el punto de vista inverso, la corrupción sirve para evitar los costos de la burocracia excesiva; los accidentes de tránsito tienen como contrapartida la posibilidad de desplazarnos más rápidamente; la discriminación implica nuestra libertad para elegir con quienes relacionarnos; $y$, la enfermedad, la posibilidad de realizar actividades que nos generan placer.

Hasta aquí, es fácil percibir como la reducción de estos "males" sociales trae aparejado el costo de reducir también la existencia de "bienes" sociales; además del costo asociado a su implementación y efectos, tanto implícitos como ocultos. A falta de información acerca de las preferencias de las personas, el trade-off que se hace entre bienes y males sociales nos lleva a exactamente la misma situación en términos de costos. Es decir, el incremento de salud, debería ser equivalente a la disminución de placer o alimentación.

Traducción libre de:

«The reality is that people who don't have enough money (or the utilities and storage) to buy and prepare decent food in decent quantities cannot (and should not) be arsed to worry about the finer nuances of nutrition. Because getting enough to eat is always our first priority. That's why Ellyn Satter (yes, her again) created the Hierarchy of Food Needs. Which looks like this: [...]. The idea is that, before we worry about nutrition (i.e., "instrumental food") we've first got to HAVE food. Enough of it. Consistently. And it's got to be acceptable to us (which, for some people, might mean not coming from the garbage, or meeting certain standards of preparation) and it's got to taste reasonably good. A little variety is nice, too». Tomado del blog A Fat Nutritionist.

Disponible en: http://www.fatnutritionist.com/index.php/if-only-poor-people-understoodnutrition/ 
Esto nos trae varias preguntas: ¿Cuál es la combinación "deseable" de placer y salud que debe tener una sociedad? ¿Quién debe elegir esa combinación? ¿En qué situaciones -en caso se considere que la respuesta a la pregunta anterior no siempre es la misma- debe ser uno u otro? En caso se considere que el nivel deseable es el "óptimo", ¿cuál es la mejor manera de llegar a dicho nivel? En caso se considere que el criterio es distinto al "óptimo", ¿cómo se establece la manera de llegar a dicho, otro, criterio?

Un error común, incluso entre famosos profesores de regulación económica, es pensar que el nivel óptimo de un "mal" social es cero. En este sentido, se ha dicho que «Muchos académicos han dicho que, bajo el modelo de Becker, en el largo plazo, los mercados competitivos deberían eliminar a las empresas que tienen una preferencia por la discriminación. En 1987, Posner, por ejemplo, dijo que la discriminación solo puede persistir si hay algún tipo de falla de mercado» ${ }^{6}$. Sumándose a la estrella del análisis económico del Derecho, Richard Posner, otro famoso profesor, esta vez de Harvard, Cass Sunstein ${ }^{7}$ (1991), ha sostenido que es un acertijo el porqué, si los mercados "sancionan" a los discriminadores, la discriminación persiste. Realmente es nada parecido a un acertijo: el mercado solo "disciplina" la discriminación, cosa que no tiene en absoluto relación con "desaparecer" la discriminación.

Si el nivel es cero -y se entiende que el mercado debería tender hacia dicho nivel-; la constatación de que el nivel de una actividad (dañina) está lejos de cero, implica que el mercado ha fallado. Nótese que no estamos hablando de una "falla de mercado", sino de una "falla de/mercado", en general. Sea como fuere, esta falla justificaría la intervención del Estado a través de prohibiciones o incentivos.

Conforme se puede desprender de los visto hasta aquí, el nivel óptimo de una actividad no necesariamente -si acaso alguna vez- es cero. Dado que el nivel óptimo de una actividad depende de la función de utilidad de cada persona, es imposible -por lo menos hasta el momento- llegar a un número exacto que

$6 \quad$ Stewart J.Schwab, Employment Discrimination. En: Boudewijn Bouckaert y Gerrit De Geest (editores). Encyclopedia of Law \& Economics. 1999. Página 576. Disponible online en: http://encyclo.findlaw.com/

Traducción libre de: «Many scholars have asserted that, under Becker's model, in the long run competitive markets will eliminate firms with a taste for discrimination. Posner (1987), for example, has said that discrimination can persist only with some kind of market failure».

$7 \quad$ Cass Sunstein. Why Markets Don't Stop Discrimination? En: Social Philosophy and Policy 2. 1991. pp 22-37. 
equivalga al nivel óptimo, a priori. Lo que se hace es suponer que si las personas pueden elegir cuánto realizar de una determinada actividad, están realizando el nivel óptimo de la actividad. Así, el nivel óptimo sería el equivalente al nivel que la sociedad está dispuesta a pagar.

Si una persona tiene libertad de elección, es decir, no existen fallas de mercado (como por ejemplo, "bienes públicos" o "externalidades"), ni problemas de razonamiento graves, una persona debería poder elegir la combinación de bienes que desee. Viceversa, cuando sí existen esos problemas, suponemos que las personas no necesariamente han escogido el nivel de actividad que los hace más "felices". Dado que en todos los casos algunas fallas de mercado existen o admitimos que las personas siempre tienen problemas de racionalidad; debemos determinar cuándo estas fallas son suficientemente grandes para justificar la regulación.

Podemos decir, entonces, que dudamos acerca de que exista una buena justificación para crear una norma como la Ley de Alimentación Saludable.

\section{¿Puede la Ley de Alimentación Saludable lograr sus propósitos?}

Asumamos que el objetivo de esta Ley es reducir la cantidad de personas especialmente niños y adolescentes- obesos en la sociedad. No existe prueba alguna acerca de esto. Primero, no sabemos la causa del sobrepeso o la obesidad de las personas en nuestra sociedad. No sabemos, tampoco, cuál es el impacto de la publicidad en el consumo de comida poco saludable incluida en el ámbito de aplicación de la Ley. El proyecto de ley -que finalmente fue aprobado- no hacía otra cosa que mostrarnos una serie de cifras absolutas como el porcentaje de niños con sobrepeso y la cantidad de dólares invertidos en publicidad de comida poco saludable en Perú.

Existe alguna evidencia ${ }^{8}$ de un efecto moderado de la publicidad, que podría reducir en $1 \%$ aproximadamente la cantidad de personas con sobrepeso. Pero esto es en EE.UU., donde existe mucha más comida chatarra y publicidad. Por lo tanto, podríamos esperar que el efecto sea aun menor en Perú.

\section{¿Existen alternativas menos gravosas?}

Shin-Yi Chou,; Inas Rashad y Michael Grossman. Fast-food restaurant advertising on television and its influence on childhood obesity. Working Paper 11879. 2005. Disponible online en: http://www.nber.org/papers/w11879 
La publicidad tiene efectos en la competencia, en el precio y la calidad de los bienes ${ }^{9}$. Un mercado con publicidad, es un mercado donde existe la competencia entre empresas y -por lo tanto- los precios tenderán a ser menos altos y dispersos ${ }^{10}$. La publicidad hace todo esto porque -aunque la intención de las empresas es persuadir a los consumidores- informa acerca de características centrales de los productos. Por ejemplo, informa que existen. También informa que ese producto es lo suficientemente bueno como para justificar determinada inversión en publicidad. Podrá parecer poco, pero es bastante.

Muchas veces los críticos de la publicidad dicen que ésta da muy poca información. Pero esto es erróneo. La publicidad es un bien accesorio a los productos que vende y -por lo tanto- es demanda al mismo tiempo que dichos productos, viéndose reflejada dicha demanda en el precio del bien vendido. En un mercado con pocas fallas, el nivel de publicidad debería tender a ser el óptimo. Es decir, la sociedad demanda exactamente la cantidad de información que quiere. A veces los políticos tienen otras ideas y ahí es donde aparecen letritas chiquitas corriendo al final de los comerciales o voces hablando a 1000 $\mathrm{km} / \mathrm{h}$.

Dados estos grandes y tangibles beneficios de la publicidad, se convierte en relevante la pregunta de si existe una alternativa para lograr el mismo propósito de reducir la cantidad de niños obesos, sin restringirla. Los impuestos selectivos al consumo de determinados bienes tienen el mismo efecto sobre el precio que las restricciones a la publicidad. Sin embargo, los impuestos generan menos distorsiones en la competencia.

Por tanto, esta norma tampoco cumple con el requisito de ser la alternativa menos gravosa para lograr su propósito.

\section{¿Tiene esta norma menos costos que beneficios?}

La norma reduce la cantidad de información en la sociedad, reduce la competencia, disminuye la calidad y aumenta los precios de los productos. Todo esto, como ya se dijo, podría llevar a una reducción en la ingesta de comida poco saludable. Esto, a su vez, puede reducir los gastos en salud de dichas personas.

9 George Stigler. The Economic of Information. En: Journal of Political Economy 3. Volumen 69. Junio 1961. Páginas 213-225. 
Hasta ahí, parece que los costos y beneficios solo se compensan. Todos preferimos combinaciones diferentes de salud/placer; así como tenemos preferencias distintas respecto a la cantidad de horas que queremos trabajar, versus la cantidad de horas que queremos dormir. No necesariamente obligarte o incentivarte a trabajar más te pondrá en una mejor situación, desde que tu combinación ideal de sueño/trabajo es distinta.

¿Por qué esta norma asume que una combinación distinta de salud/placer hará más feliz -o "mejor" en cualquier otro sentido- a la sociedad? No se sabe.

\section{Conclusiones}

Muchas veces desconfiamos de la capacidad de las personas para tomar sus propias decisiones basados en supuestos problemas de racionalidad. Esto, sin embargo, contrasta con nuestra confianza por otras personas -éstas con cargos públicos- que nos prueban día a día su irracionalidad.

Esta norma ha sido dictada sin tener alguna evidencia sobre si es necesario dictarla; o sus efectos; o si existe una mejor alternativa; o si consigue más beneficios que costos a la sociedad. Desde mi punto de vista, esto la convierte es una norma completamente irracional.

El problema es que es una irracionalidad con efectos para todos. Cuando una persona decide comer de más; solo se "afecta" -si acaso- a ella misma. Cuando un congresista decide hacer una norma irracional, afecta a todo el país. 